\title{
THE DESIGN OF A DYNAMIC TEMPERATURE FIELD MEASUREMENT SYSTEM IN CVT GEARBOX TRACTOR
}

\author{
Maohua Xiao ${ }^{1}$, Yinchu Yang ${ }^{1}$, Petr Bartos ${ }^{2}$, Andrea Bohata ${ }^{2}$, Kailing Chen $^{1}$ \\ ${ }^{1}$ College of Engineering, Nanjing Agriculture University, Nanjing 210031, China \\ ${ }^{2}$ Faculty of Agriculture, University of South Bohemia, Studentska 1668, Czech
}

\begin{abstract}
CVT tractor has the characteristics of high-energy consumption and low-energy efficiency, which makes the monitoring of its energy consumption significant. According to the characteristics of the CVT tractor, a high-speed and high-precision temperature measurement system was built in this article. By the late experimental demonstration and data processing, the temperature field was simulated by the simulation of the ANSYS and the analysis of Matlab. This system was used to realize the measurement of temperatures with a microcontroller, which mainly included a AT89S52 microcontroller, temperature sensors, a keyboard and a display circuit. It can accomplish the measurement of the temperatures from eight different directions in real time, and it can display and set temperatures on the LCD screen as well. When the real-time temperature was higher than the temperature set before, the buzzer would alert automatically. The late tests showed that the dynamic temperature field measurement system had the advantages of being convenient and practical, which can not only measure the temperature of the CVT tractor, but also measure temperatures of various kinds of dynamic temperature fields after some specific improvements. The temperature measurement system has potentials in various kinds of fields if it was developed to meet the need of humans.
\end{abstract}

Keywords: Temperature Field, Measurement, SCM, ANSYS Simulation, MATLAB Analysis.

\section{Introduction}

Agricultural tractor, one kind of typical agricultural mechanics, has low energy efficiency because of its poor working conditions. When the tractors work, how to increase the utilizing rate to the maximum and reduce the consumption of useless work is meaningful to improve the energy efficiency of the tractor and develop the low-carbon economy. In order to improve the problem of high-energy consumption, many methods were proposed for the tractor. In order to minimize the time and fuel, Tomas Nilsson developed a method of a short loading cycle with a CVT wheel loader[1]. Young-Jun Park developed a network model of a Hydromechanical transmission(HMT) for tractors and found that the network model was suitable for analyzing the power transmission characteristics of HMT after development[2]. To assess fuel consumption efficiency, Marcelo Silveira de Farias researched the factors of travel speed and load levels ${ }^{[3]}$. Xiao Maohua designed a new type of hydromechanical continuously variable transmission (HMCVT) and the new tractor's fuel economy was improved through the experimental verification ${ }^{[4]}$.

Further, the detection and control technologies of temperature were widely used in industrial production and daily life ${ }^{[5]}$. M. V. Mekhrengin developed a spectral system for the temperature measurement of gas flow inside the combustion chamber ${ }^{[6]}$. Xue-jun Liu proposed an adaptive method for the distributed temperature sensor to eliminate the influence of fiber attenuation and APD dark current fluctuation[7]. There were a thermocouple temperature measurement device and thermal resistance in traditional temperature measurement system, but the output of thermocouple and RTD was analog.

Generally, it required some external hardware circuit before the output was converted to a readable digital controller ${ }^{[8]}$. The design and the establishing of a digital temperature sensor DS18B20 in this experiment was realized by a SRC89C52 microcontroller and the basic temperature measurement system was introduced in this article. Though the mathematical software of ANSYS and MATLAB, the dynamic temperature field measurement system was simulated and examined.

\section{The Establishing of the Temperature Measurement System}

STC89C52 microcontroller was applied as a control unit for collecting temperature information [9] by DS18B20 temperature sensors.

When the temperature is higher than the set temperature, the buzzer would alert automatically. 
In this way, MCU reflected its advantages of simple structure, easy operation and convenient control. And this MCU had powerful bit manipulation instructions, of whose I/O port could address by bit and the program space could reach up to $8 \mathrm{~KB}$, which was adequate for the design in this paper. In order to realize self-control, the information was sent into the main control unit to complete the appropriate action after the data processed. The LCD screen displayed the real-time working conditions by 1602 LCD for its feature of flexibility and clearness to read. The method using software to solve the complex hardware circuit part[10] was simplified for the system so that it could realize all kinds of features and was easy to satisfy the specific requirement of this subject.

The main components of the system included AT89S52 microcontroller, temperature sensor, the keyboard, and the display circuit. Fig.1 below showed the circuit's overall scheme of the temperature measurement system. And the main control circuit of the system was shown in Fig. 2.

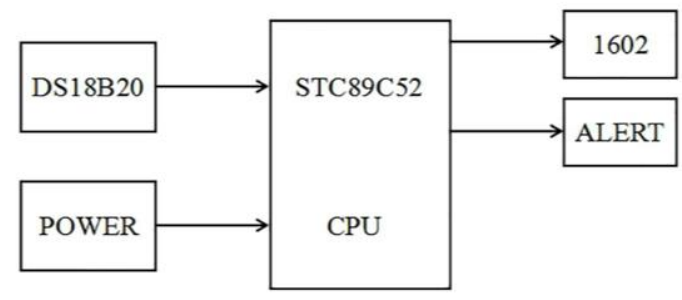

Figure 1: The overall scheme of the circuit

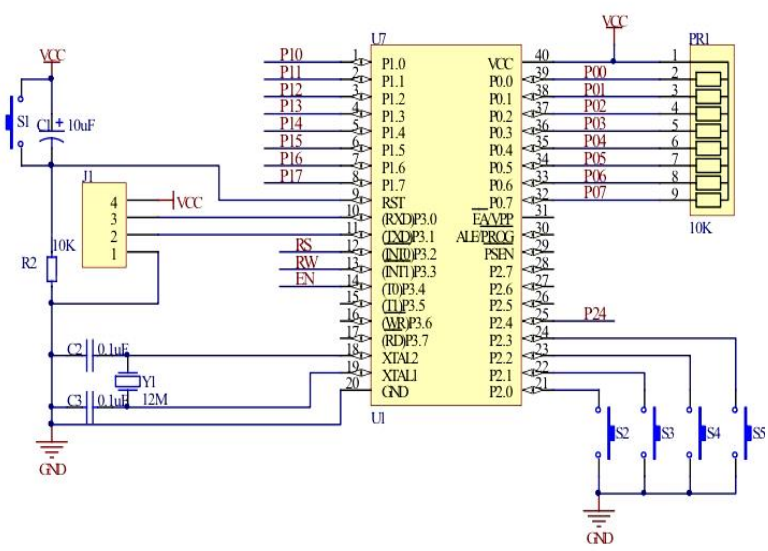

Figure 2: The main control circuit

Hardwares of the system consisted of the main control module, the power module, the display module, the SCM least operating system, the temperature sensors, the buzzer, the light-emitting diode alarm module. Softwares consisted of the main program module and each subroutine modules. The main control circuits needed to be welded according to the overall design and SCM procedures. And the temperature measurement system established was shown in Fig. 3.

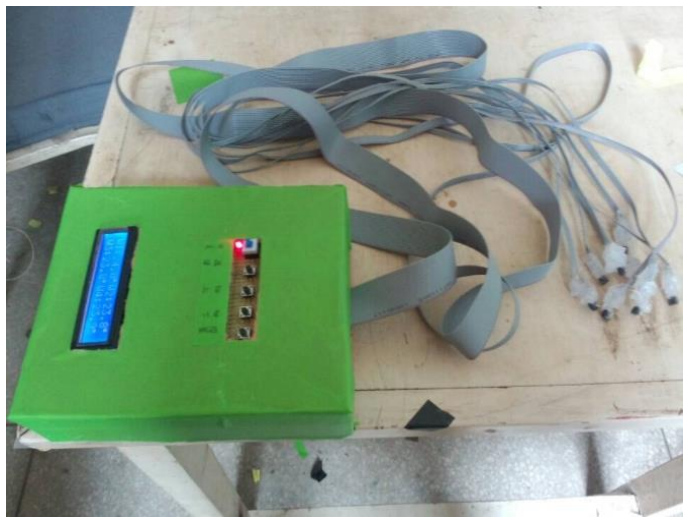

Figure 3: The temperature measurement system

\section{Experiment and Data Processing}

\subsection{The experiment of temperature field of a plane in space}

In order to simulate the temperature field, a small water tank was applied as the experimental apparatus. The small tank was filled with water, and a heat source device was put into it for simulating an exothermic condition in this transmission, which could realize the simulation of transverse temperature in CVT. The 12 temperature sensors were all immersed into water and distributed on a plane of the tank uniformly. The temperatures of the 12 points were measured on this plane and the corresponding data was collected and analyzed to simulate the temperature field of the plane. Fig.4 displayed the distribution of the temperature sensors.

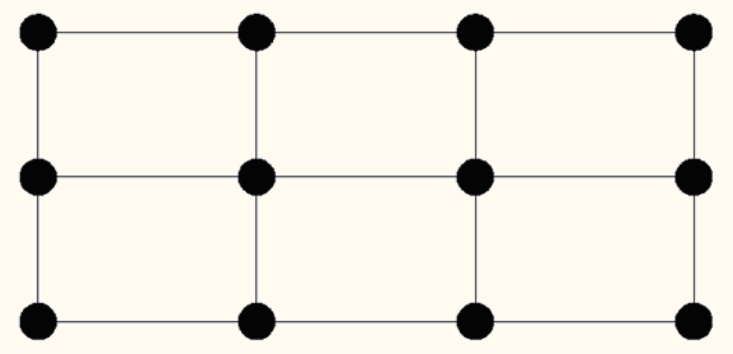

Figure 4: The distribution of temperature sensors

The data was measured with an interval of 10 seconds, and Table 1 presented the 10 sets of the data of the temperatures measured. In order to verify the variable regulation of the data with the time change, the ANSYS simulation was used for the experimental model. Assumed conditions in the ANSYS were set as follows: the initial temperature of the water was $25^{\circ} \mathrm{C}$, the thermal conductivity of water was $0.608 \mathrm{~W} /(\mathrm{m} * \mathrm{~K})$, the surface coefficient of heat transfer was $100 \mathrm{~W} /\left(\mathrm{m}^{2} * \mathrm{~K}\right)$, the heating temperature of the heating source was $400^{\circ} \mathrm{C}$, the density of water was $1000 \mathrm{Kg} / \mathrm{m}^{3}$, and the specific heat capacity was $4200 \mathrm{~J} /\left(\mathrm{kg} *{ }^{\circ} \mathrm{C}\right)[11]$. 
The ANSYS simulation results were displayed in Fig. 5. And the temperature field of the tank plane and isotherm of the tank plane were simulated by the software of Matlab and the results of the simulation were presented in Fig. 6 and Fig. 7, respectively.

Table 1. The data of the temperatures

\begin{tabular}{|c|c|c|c|c|c|c|c|c|c|c|c|c|}
\hline time & 1 & 2 & 3 & 4 & 5 & 6 & 7 & 8 & 9 & 10 & 11 & 12 \\
\hline $10 \mathrm{~s}$ & 46.1 & 46.4 & 45.5 & 41.8 & 43.1 & 46.8 & 46.9 & 45.7 & 47.3 & 47.1 & 46.0 & 43.2 \\
\hline $20 \mathrm{~s}$ & 46.3 & 46.5 & 45.7 & 42.1 & 43.3 & 47.0 & 47.2 & 46.0 & 47.6 & 47.3 & 46.3 & 43.6 \\
\hline $30 \mathrm{~s}$ & 46.6 & 47.0 & 45.9 & 42.3 & 43.5 & 47.1 & 47.5 & 46.2 & 47.8 & 47.5 & 46.5 & 43.8 \\
\hline $40 \mathrm{~s}$ & 46.9 & 47.2 & 46.4 & 42.5 & 43.6 & 47.4 & 47.6 & 46.5 & 48.1 & 47.6 & 46.6 & 44.1 \\
\hline $50 \mathrm{~s}$ & 47.3 & 47.6 & 47.1 & 42.7 & 44.2 & 48.0 & 48.5 & 47.0 & 48.8 & 48.3 & 47.1 & 44.6 \\
\hline $60 \mathrm{~s}$ & 47.6 & 49.9 & 47.2 & 43.0 & 44.3 & 48.1 & 48.6 & 47.1 & 49.0 & 48.5 & 47.4 & 44.8 \\
\hline $70 \mathrm{~s}$ & 47.9 & 48.0 & 47.7 & 43.2 & 44.4 & 48.5 & 49.0 & 47.6 & 49.3 & 48.9 & 47.6 & 45.5 \\
\hline $80 \mathrm{~s}$ & 48.0 & 48.3 & 47.8 & 43.6 & 44.6 & 48.6 & 49.1 & 47.8 & 49.4 & 49.0 & 47.7 & 45.1 \\
\hline $90 \mathrm{~s}$ & 48.4 & 48.6 & 48.3 & 44.0 & 45.1 & 49.0 & 49.6 & 48.3 & 49.9 & 49.5 & 48.4 & 45.5 \\
\hline $100 \mathrm{~s}$ & 48.6 & 48.7 & 48.4 & 44.3 & 45.4 & 49.3 & 49.9 & 48.5 & 50.1 & 49.7 & 48.2 & 45.8 \\
\hline
\end{tabular}

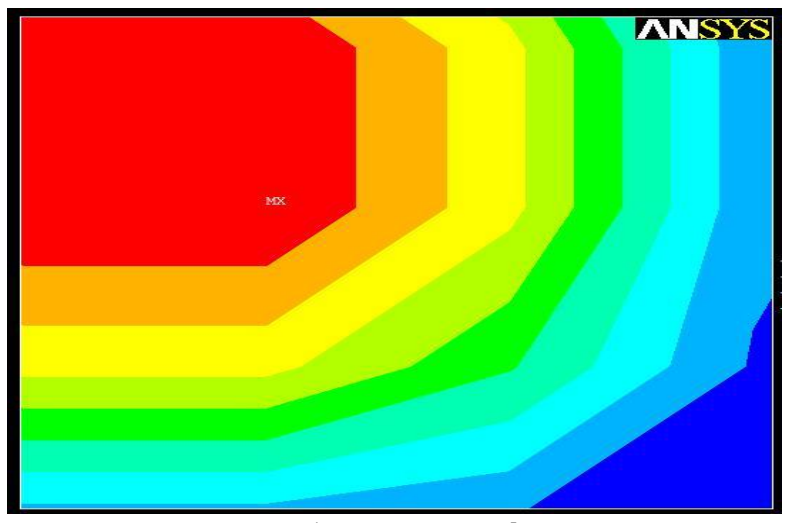

Figure 5: ANSYS simulation

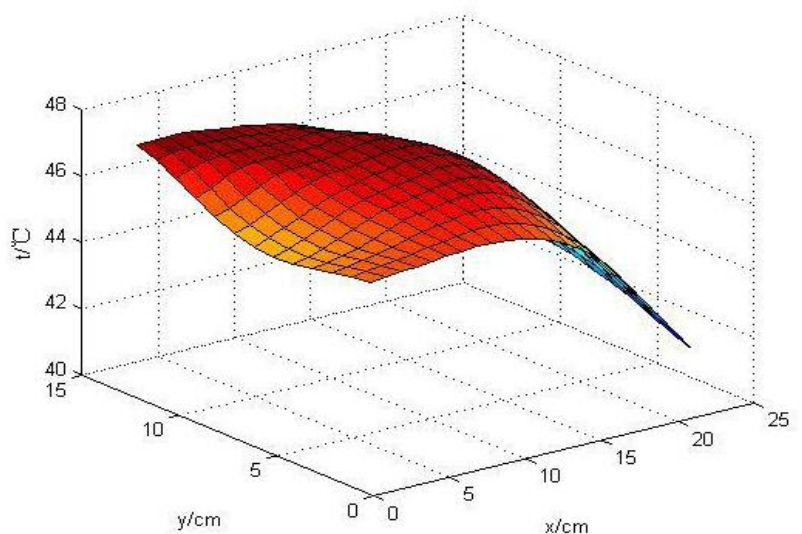

Figure 6: Temperature field of the tank plane 


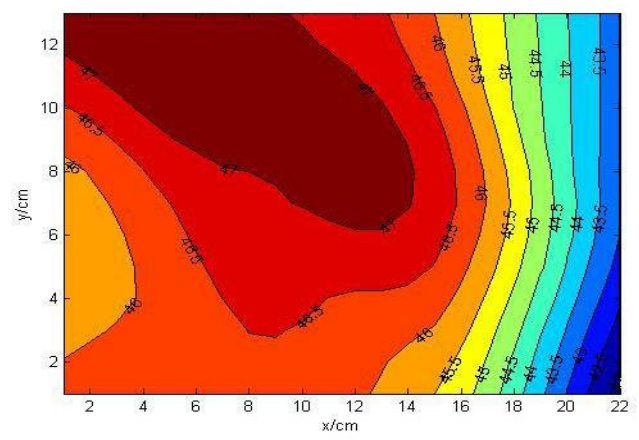

Figure 7: Isotherm of the tank plane

Through the simulation of the experimental data with the ANSYS as well as Matlab, it can be inferred that the experimental results were in line with the simulating results.

\subsection{The experiments of temperature field in space}

In the following experiments, the tank was filled with hot water in a half, and then the 12 temperature sensors were distributed on a plane of the box uniformly, which was tilted with an angle of 45 relative to horizontal plane so that half of the sensors were immersed in the water and the rest were exposed to the air[12].

The temperatures of 12 points were measured on this plane and the underwater temperatures as well as overwater temperatures were obtained. In this way, it could realize the simulation of the vertical temperature field of the CVT.

Table 2 presented the experimental results of the temperature field in space with the intervals of 10 seconds.

The simulation of the temperature field of the tank by the software of Matlab was shown as Fig. 8 and Fig. 9.

Table 2. The experimental results of the temperature field

\begin{tabular}{|c|c|c|c|c|c|c|c|c|c|c|c|c|}
\hline time & 1 & 2 & 3 & 4 & 5 & 6 & 7 & 8 & 9 & 10 & 11 & 12 \\
\hline $10 \mathrm{~s}$ & 41.7 & 42.5 & 29.7 & 28.9 & 42.6 & 40.1 & 25.3 & 25.5 & 42.1 & 41.8 & 35.4 & 26 \\
\hline $20 \mathrm{~s}$ & 41.8 & 42.4 & 29.5 & 28.6 & 42.6 & 40.1 & 25.4 & 25.5 & 42.1 & 41.8 & 34.6 & 25.8 \\
\hline $30 \mathrm{~s}$ & 42.0 & 41.5 & 29.1 & 28.6 & 42.7 & 40.2 & 25.1 & 25.4 & 42.1 & 41.8 & 33.1 & 25.7 \\
\hline $40 \mathrm{~s}$ & 41.7 & 41.1 & 29.1 & 28.5 & 42.6 & 40.1 & 24.9 & 25.3 & 42.1 & 42.0 & 30.8 & 25.7 \\
\hline $50 \mathrm{~s}$ & 41.8 & 41.0 & 29.2 & 28.4 & 42.6 & 40.1 & 25.1 & 25.7 & 42.1 & 41.8 & 30.5 & 26.0 \\
\hline $60 \mathrm{~s}$ & 41.6 & 40.9 & 29.2 & 28.3 & 42.6 & 40.2 & 25.1 & 25.8 & 42.1 & 41.8 & 29.0 & 26.3 \\
\hline $70 \mathrm{~s}$ & 41.5 & 40.8 & 28.9 & 28.3 & 42.5 & 40.3 & 25.0 & 26.7 & 42.1 & 41.8 & 29.3 & 26.3 \\
\hline $80 \mathrm{~s}$ & 41.5 & 40.7 & 28.9 & 28.3 & 42.5 & 40.3 & 25.0 & 26.7 & 42.1 & 41.8 & 29.3 & 26.3 \\
\hline $90 \mathrm{~s}$ & 41.6 & 40.6 & 28.6 & 28.2 & 42.5 & 40.1 & 25.4 & 26.4 & 42.0 & 41.8 & 29.0 & 27.1 \\
\hline $100 \mathrm{~s}$ & 41.6 & 40.5 & 29.0 & 28.3 & 42.5 & 40.1 & 25.6 & 26.3 & 42.0 & 41.8 & 29.0 & 27.1 \\
\hline
\end{tabular}

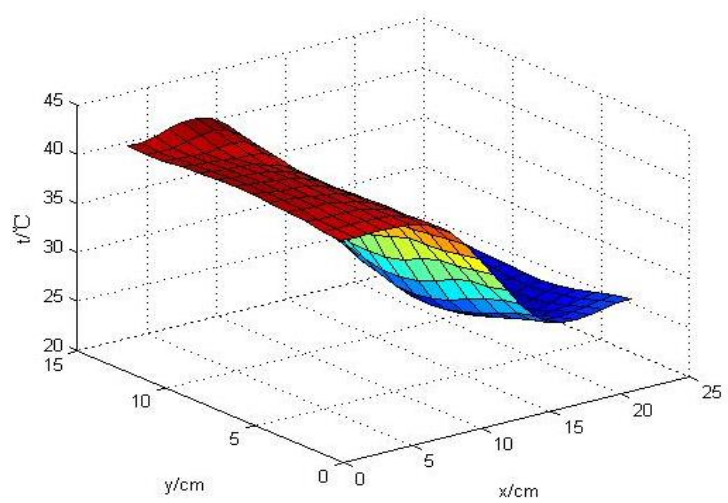

Figure 8: Vertical temperature field of the tank 


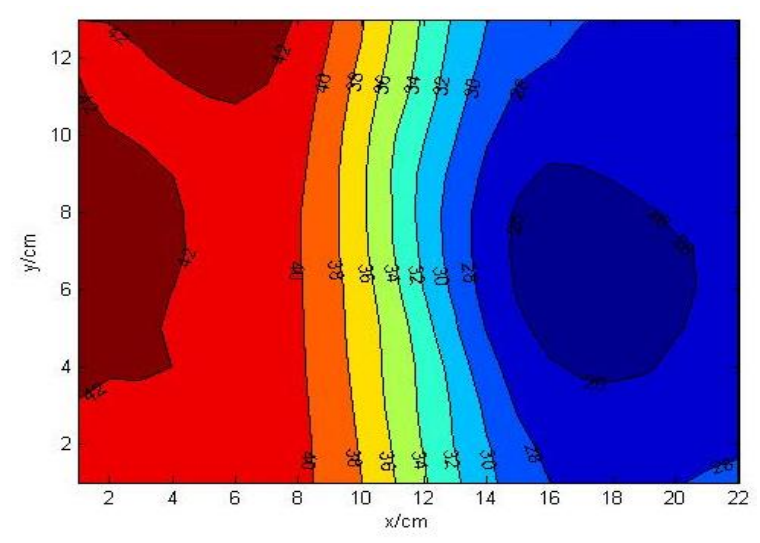

Figure 9: Isotherm of the vertical surface

Through the simulation experiment, it could also be inferred that the experimental results were in line with the simulating results in the condition of space.

\subsection{The temperature conditions of each point}

Fig. 10 (horizontal analog) and Fig. 11 (vertical analog) showed the temperature changes over time of each measurement point obtained from two experiments' data.

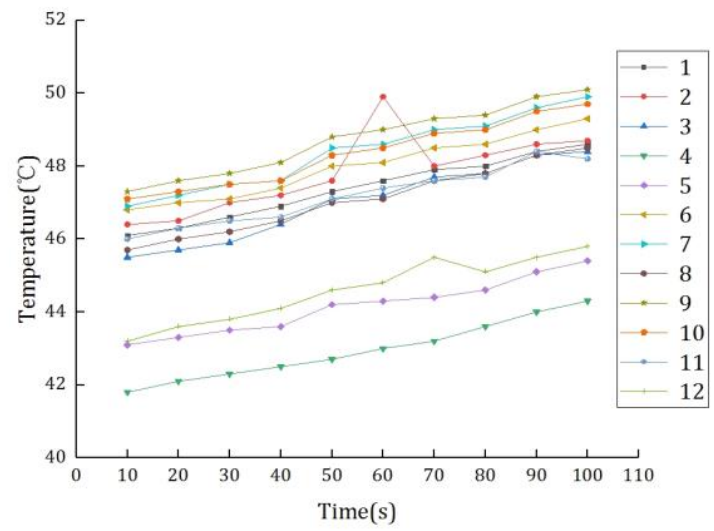

Fig 10: Temperature changes over time of each point on the horizontal plane

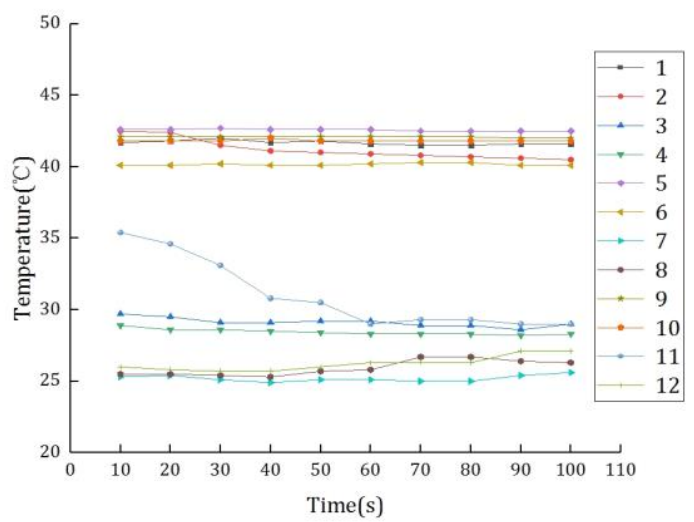

Figure 11: Temperature changes over time of each point on the vertical plane
The continuous changes of the temperatures over time of each point could be measured by the system and reflected by the LCD display when the real-time temperature was out of the range of the temperature that was predetermined. When the real-time temperature was higher or lower than the set temperature to a certain extent, the buzzer would alarm to warn the users of the inappropriate working conditions. It could be inferred from the experiment that the detection and the prevention of the gearbox had the practical and significant influence in the practical application, especially when the temperature of the gearbox went blinking.

\section{Conclusions}

This temperature measurement system possessed multifunctions, including the acquisition of the temperature signals, the display of the temperature, simple man-machine conversation and alarming when abnormal temperature happened, which was appropriate for the measurement of a temperature field of CVT. Although this system was mainly designed for the collection, the display and monitoring of the measured data, which made it lack the function of the control and regulation, it could be further developed both in the fields of hardwares and softwares.

When the hardware was developed, the control functions of the system would be extended through software programming to adapt the measured data of the environmental temperature, the humidity and the flux, etc to satisfy the need of human. Nowadays, with the development of high automation in the industry and agriculture, the automatic monitoring of the environment has aroused wide attention from the public.

The temperature measurement system designed in this paper could hold a huge market and wide application prospects.

\section{Acknowledgements}

This research is based upon work supported by the Jiangsu Science and Technology Support Program (BE2018127).

\section{References}

[1] Tomas Nilsson, Anders FroBerg, Jan Aslund. Fuel and time minimization in a CVT wheel loader application [J]. IFAC Proceedings Volumes, 2013, 46(21): 201-206.

[2] Park Y J, Kim S C, Kim J G. Analysis and verification of power transmission characteristics of the hydromechanical transmission for agricultural tractors [J]. Journal of Mechanical Science and Technology, 2016, 30(11): 5063-5072. 
[3] De F M S, Schlosser José Fernando, Pilar L, et al. Fuel consumption efficiency of an agricultural tractor equipped with continuously variable transmission [J]. Ciência Rural, 2017, 47(6): 201206.

[4] Xiao M H, Zhao J, Wang Y W, Zhang H J, Lu Z X, Wei W H. Fuel economy of multiple conditions self-adaptive tractors with hydro-mechanical CVT[J]. Int J Agric \& Biol Eng, 2018, 11(3): 102109.

[5] Sha Zhanyou. Principles and applications of intelligent integrated temperature sensor [D]. Mechanical Industry Press, 2002.

[6] M.V. Mekhrengin, I.K. Meshkovskii, et al. Multispectral pyrometer for high temperature measurements inside combustion chamber of gas turbine engines [J]. Measurement 2019, (139): 355-360.

[7] Xue-Jun L, Hao L, Bi-Xian Y, et al. A double-ended Raman temperature measurement method for hazardous chemicals warehouse [J]. Optik, 2018, (169): 62-68.
[8] Hao Lihong, Lin Ling, Yi Zhiming, Li Gang. Issues of thermocouple [J]. Test and measurement and sensing. 2003. (1): 83-85.

[9] Kwofie S. Plasticity model for simulation, description and evaluation of vibratory stress relief [J]. Materials Science \& Engineering: A (Structural Materials: Properties, Microstructure and Processing), 2009, 516(1-2): 154-161.

[10] Lee J H, Kalkan I. Analysis of Thermal Environmental Effects on Precast, Prestressed Concrete Bridge Girders: Temperature Differentials and Thermal Deformations [J]. Screen, 2012, 47(1): 129-132.

[11] Brown, A. A physical model of the atmospheric aerosol turbidity for estimating the illuminance of direct sunlight [J]. Lighting Research and Technology, 2008, 40(4): 347-358.

[12] Schindler A K, Ruiz J M, Rasmussen R O, et al. Concrete pavement temperature prediction and case studies with the FHWA HIPERPAV models [J]. Cement and Concrete Composites, 2004, 26(5): 463-471. 